\title{
Pathological prognostic factors predicting lymph node metastasis in submucosal invasive (T1) colorectal carcinoma
}

\author{
Yoko Tateishi ${ }^{1,4}$, Yukihiro Nakanishi ${ }^{2,5}$, Hirokazu Taniguchi ${ }^{1}$, Tadakazu Shimoda ${ }^{1}$ and \\ Satoshi Umemura ${ }^{3}$ \\ ${ }^{1}$ Clinical Laboratory Division, National Cancer Center Hospital and Research Institute, Tokyo, Japan; \\ ${ }^{2}$ Pathology Division, National Cancer Center Hospital and Research Institute, Tokyo, Japan and ${ }^{3}$ Department \\ of Medical Science and Cardio-Renal Medicine, Yokohama City University Graduate School of Medicine,
} Kanagawa, Japan

\begin{abstract}
Lymph node metastasis occurs in as many as $16 \%$ of patients with submucosal invasive colorectal carcinoma. We investigated the association between histopathological factors and lymph node metastases in 322 consecutive patients with submucosal invasive colorectal carcinoma who had undergone radical colectomy with lymph node dissection to detect patients at high risk of lymph node metastasis without measuring the depth of submucosal invasion. Lymph node metastasis was found in $46(14.3 \%)$ of 322 patients with submucosal invasive colorectal carcinoma. Univariate analysis showed that each of the following histopathological factors had a significant influence on lymph node metastasis: invasion depth, lymphatic invasion, venous invasion, tumor differentiation, growth pattern of the intramucosal tumor component, complete disruption of the muscularis mucosa due to tumor invasion, and tumor budding at the submucosal invasive front. Multivariate analysis showed that lymphatic invasion $(P<0.01)$, tumor differentiation $(P<0.01)$, and tumor budding $(P<0.01)$ were significantly associated with lymph node metastasis. All 46 cases of lymph node metastasis showed at least one of the following findings: lymphatic invasion, moderately or poorly differentiated tumor grade, tumor budding, or complete disruption of the muscularis mucosa due to tumor invasion. Patients with submucosal invasive colorectal carcinoma that show at least one of three factorslymphatic invasion, moderately or poorly differentiated tumor grade, or tumor budding -are at high risk for lymph node metastasis. All of the patients with lymph node metastasis, who did not have any of these factors, showed a completely disrupted muscularis mucosa.
\end{abstract}

Modern Pathology (2010) 23, 1068-1072; doi:10.1038/modpathol.2010.88; published online 14 May 2010

Keywords: colorectal carcinoma; submucosal invasive carcinoma; lymph node metastasis; histopathological study; endoscopic mucosal resection

Up to $16 \%$ of patients with submucosal invasive (T1) colorectal carcinoma are reported to have lymph node metastasis, whereas lymph node metastasis is

Correspondence: Dr T Shimoda, MD, PhD, Clinical Laboratory Division, National Cancer Center Hospital, 5-1-1 Tsukiji, Chuo-ku, Tokyo 104-0045, Japan.

E-mail: tshimoda@ncc.go.jp

Presented at a meeting of the 15th United European Gastroenterology Week, Paris, France, October 2007.

${ }^{4}$ Current address: Department of pathology, Tokyo Metropolitan Komagome Hospital, Tokyo, Japan.

${ }^{5}$ Current address: Department of pathology, St Louis University Medical Center, St Louis, MO, USA.

Received 29 June 2009; revised 22 February 2010; accepted 1 March 2010; published online 14 May 2010 reportedly absent in patients with mucosal colorectal carcinoma. ${ }^{1-7}$ Consequently, mucosal colorectal carcinoma is generally treated by endoscopic mucosal resection. ${ }^{1-7}$ Because radical colectomy with lymph node dissection is an invasive treatment, patients with submucosal invasive colorectal carcinoma without lymph node metastasis should be treated by endoscopic mucosal resection, if possible. However, few reports have proposed definite histopathological criteria for additional treatment after endoscopic mucosal resection in patients with submucosal invasive colorectal carcinoma. ${ }^{4,7}$

Many investigators have reported that lymphatic invasion, venous invasion, tumor differentiation, and the degree of submucosal invasion depth are 
risk factors of lymph node metastasis. ${ }^{1-7}$ Haggitt et $a l^{1}$ proposed a four-level classification system for pedunculated lesions with invasive carcinoma based on the depth of invasion, and considered that level 4 invasion, which means invasion of carcinoma into the submucosa to a level deeper than the stalk, to be an important clinical prognostic factor for lymph node metastasis. However, this classification is less useful for non-polypoid lesions without a stalk because all such lesions with submucosal invasion are classed as level 4. Kudo et al have suggested a three-grade classification that divides the thickness of the submucosa into three layers (sm1, sm2, or sm3), and reported that this classification is useful for predicting lymph node metastasis. ${ }^{3,8}$ However, this classification cannot be applied for analysis of endoscopic mucosal resection specimens because such specimens do not include the full thickness of the submucosal layer. Kitajima et $a l^{4}$ have reported that, in the absence of lymphatic invasion, cases of non-polypoid submucosal invasive carcinoma less than $1 \mathrm{~mm}$ in depth do not have lymph node metastasis. However, there is a problem when measuring the depth of submucosal invasion if the muscularis mucosa is completely disrupted due to tumor invasion. In such cases, there might be considerable interobserver inconsistency in measurements of submucosal invasion depth. In this study, therefore, we tried to detect patients at high risk of lymph node metastasis after endoscopic mucosal resection without measuring the depth of submucosal invasion.

\section{Materials and methods}

\section{Patients}

Our series comprised 322 consecutive patients who had undergone radical colectomy, involving complete local excision of the tumor with systemic lymphadenectomy, for treatment of submucosal invasive colorectal carcinoma at the National Cancer Center Hospital, Tokyo, Japan, between January 1992 and December 2005. Among these patients, the histopathological features of 155 have already been reported. ${ }^{6}$ The present series of cases did not include any that were treated by endoscopic mucosal resection and transanal mucosal resection; any with post-endoscopic mucosal resection recurrence; or any involving synchronous advanced cancers, familial adenomatous polyposis, or inflammatory bowel disease. The patients comprised 216 men $(67.1 \%)$ and 106 women $(32.9 \%)$ with a mean age of 61.4 years (range 30-94 years). Our series comprised 33 polypoid carcinomas and 289 sessile carcinomas. In these 322 patients, 127 tumors were located in the rectum, 99 in the left colon, and 96 in the right colon. The median number of lymph nodes removed with each surgical specimen was 14 (range 1-63 lymph nodes).

\section{Histopathological Review}

Serial 5-mm-thick tissue sections of the entire lesion were cut from resected specimens fixed with $10 \%$ buffered formalin, embedded in paraffin, and stained with hematoxylin and eosin using standard methods. The submucosal invasion depth, tumor size, lymphatic invasion, venous invasion, tumor differentiation, tumor budding at the submucosal invasive front, and the status of the muscularis mucosa were evaluated histopathologically. The submucosal invasion depth was measured using a microscope with an ocular lens scale. In specimens where the muscularis mucosa was incompletely disrupted by ulceration or tumor invasion, the muscularis mucosa level was estimated by drawing a line connecting the remaining parts of the muscularis. When the muscularis mucosa was completely disrupted due to tumor invasion, we measured the distance from the tumor surface to the invasive front. ${ }^{4,6}$ Tumor differentiation was assessed according to the World Health Organization classification (Figure 1a-c). ${ }^{9}$ The criteria used for moderate differentiation were the presence of well-formed glands with architectural complexity including a cribriform pattern. The tumor cells were at least low columnar, and the nuclei were modestly enlarged but polarity was largely maintained. The tumor shown in Figure $1 \mathrm{~b}$ is an example of one of several different patterns that are all included under the term 'moderately differentiated'. Tumor budding was defined as an isolated single cancer cell or a cluster composed of fewer than five cancer cells (Figure 2). ${ }^{10,11}$ If there is a lot of inflammation, it is not always easy to recognize tumor budding. In our series, tumor budding was defined strictly as the presence of an isolated single cancer cell or a cluster of less than five tumor cells, excluding equivocal tumor budding intermingled with inflammatory cells. The status of the muscularis mucosa was classified as type A (preserved or incompletely disrupted by tumor invasion, Figure 3) or type B (completely disrupted by tumor invasion, Figure 4). ${ }^{6}$ Independent histopathological reviews were performed by two experienced pathologists (YT and HT) who had no prior knowledge of the clinical outcomes of the patients. The results were compared, and any discrepancies were resolved by consensus at a meeting following a further histopathological review.

\section{Statistical Analysis}

Unconditional logistic analysis was performed using the JMP software package (SAS Institute) to examine the association between histological factors and lymph node metastasis. All $P$-values were twosided, and the significance level was set at $P<0.05$.

\section{Results}

Lymph node metastasis was found in 46 (14.3\%) of 322 patients with submucosal invasive colorectal carcinoma. The relationships between the histo- 

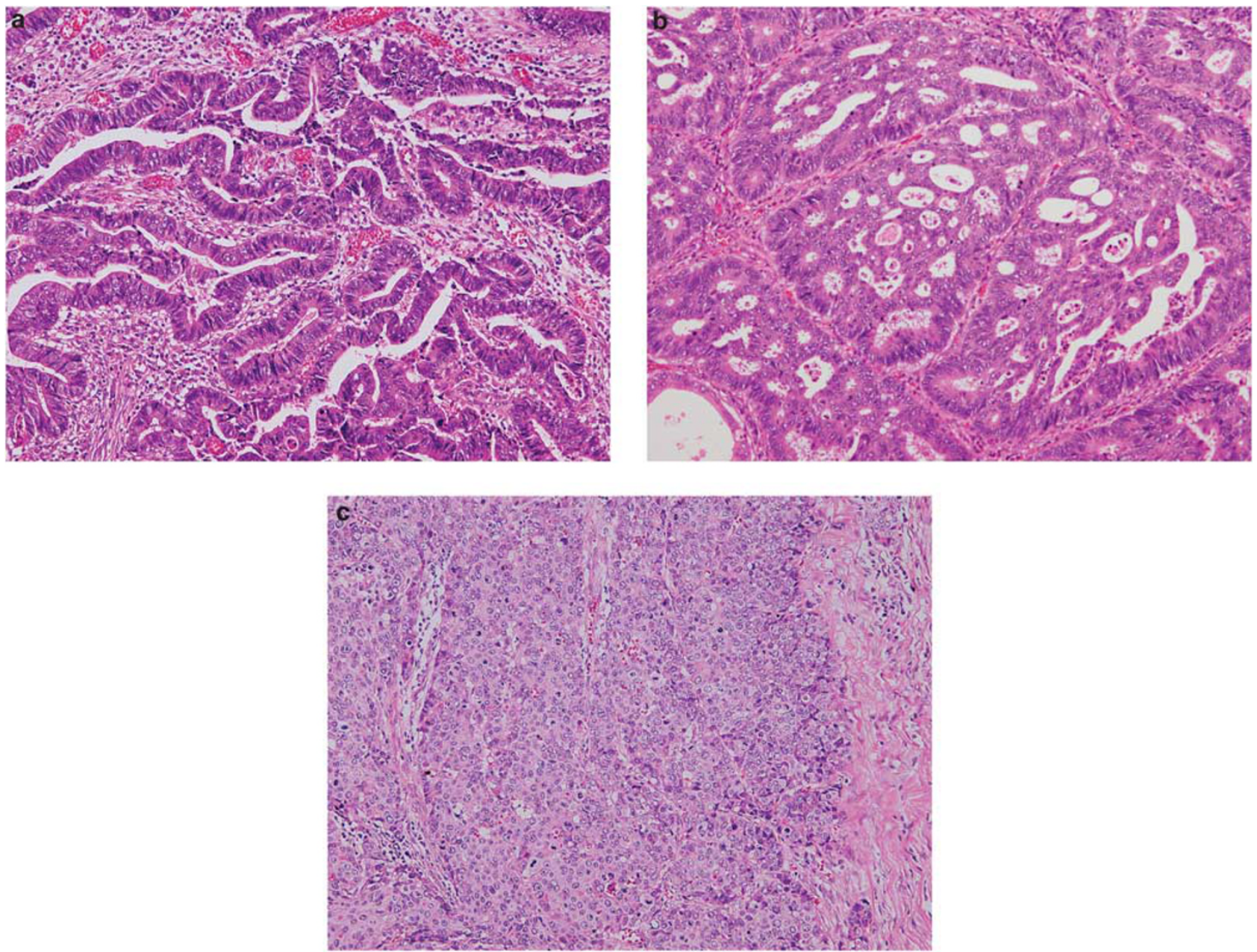

Figure 1 (a) Well-differentiated adenocarcinoma is characterized by a simple tubular structure or tree-like external branching. (b) Moderately differentiated adenocarcinoma is characterized by sieve-like internal branching and a 'cribriform pattern'. (c) Poorly differentiated adenocarcinoma is characterized by lack of gland formation.

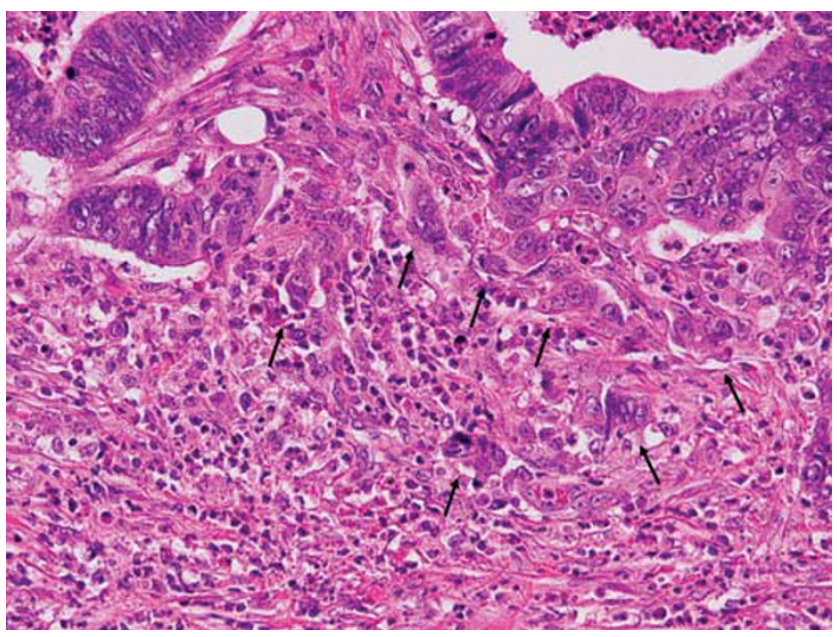

Figure 2 Tumor budding (an isolated single cancer cell or a cluster composed of fewer than five cancer cells). Arrows indicate tumor buddings.

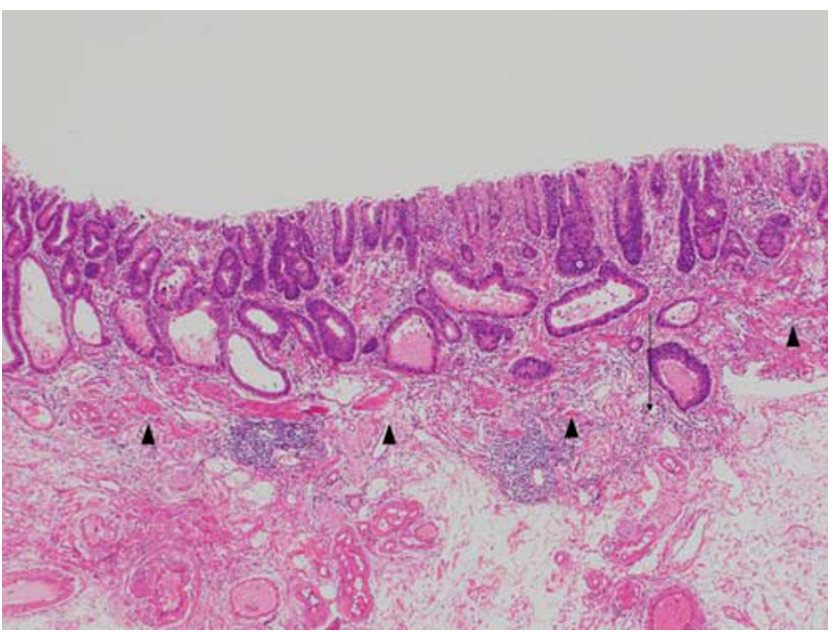

Figure 3 Although minute submucosal invasion is evident, the muscularis mucosa is incompletely disrupted and relatively well preserved, corresponding to type A muscularis mucosa. Arrows indicate submucosal invasion, and arrowheads indicate preserved areas of the muscularis. 


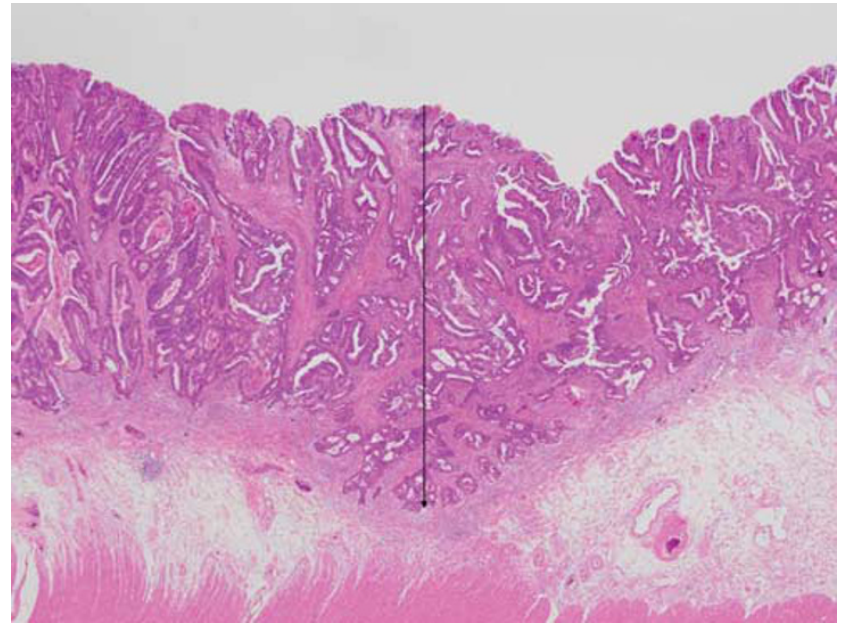

Figure 4 The muscularis mucosa is completely disrupted by tumor invasion, corresponding to type B muscularis mucosa.

pathological findings and lymph node metastases in the 322 patients were determined using univariate and multivariate analysis, and are shown in Tables 1 and 2. Univariate analysis showed that each of the following histopathological factors had a significant influence on lymph node metastasis: tumor size (10 $\mathrm{mm}$ or less vs more than $10 \mathrm{~mm}$ ), submucosal invasion depth $(1000 \mu \mathrm{m}$ or less vs more than $1000 \mu \mathrm{m}$ ), lymphatic invasion, venous invasion, tumor differentiation, the status of the muscularis mucosa (type A vs B), and tumor budding at the submucosal invasive front. Multivariate analysis revealed that lymphatic invasion, tumor differentiation, and tumor budding at the submucosal invasion front were independently correlated with lymph node metastasis. Of 46 patients with lymph node metastasis, $40(87.0 \%)$ had either moderate or poor tumor differentiation, tumor budding at the submucosal invasive front, or lymphatic invasion. The remaining six $(13.0 \%)$ showed type $\mathrm{B}$ muscularis mucosa status without any of the above three findings.

\section{Discussion}

In this study, lymph node metastasis was observed in $46(14.3 \%)$ of 322 patients with submucosal invasive colorectal carcinoma. Multivariate analysis showed that lymphatic invasion, tumor differentiation, and tumor budding were significantly associated with lymph node metastasis. Of a total of 46 cases with lymph node metastasis, 40 (87.0\%) had at least one of three factors: moderate or poor tumor differentiation, tumor budding at the submucosal invasive front, and lymphatic invasion. The remaining six cases $(13.0 \%)$ with lymph node metastasis showed type B muscularis mucosa status without any of these factors. Thus, using these four risk factors, we were able to select all of the
Table 1 Univariate analysis of relationship between histopathological predictive factors for lymph node metastasis

\begin{tabular}{|c|c|c|c|}
\hline & $\begin{array}{c}\text { No. of patients } \\
\text { with } \\
\operatorname{LNM}(\mathrm{n}=46)\end{array}$ & $\begin{array}{c}\text { No. of patients } \\
\text { without } \\
\text { LNM }(\mathrm{n}=276)\end{array}$ & $\begin{array}{c}\text { Univariate } \\
\mathrm{P} \text {-value }\end{array}$ \\
\hline \multicolumn{4}{|c|}{ Status of the muscularis mucosa } \\
\hline Type $\mathrm{A}^{\mathrm{a}}$ & $1(2 \%)$ & $40(98 \%)$ & \multirow[t]{2}{*}{0.02} \\
\hline Type $\mathrm{B}^{\mathrm{b}}$ & $45(16 \%)$ & $236(84 \%)$ & \\
\hline \multicolumn{4}{|c|}{ Submucosal invasion depth $(\mu \mathrm{m})$} \\
\hline$<1000$ & $1(3 \%)$ & $33(97 \%)$ & \multirow[t]{2}{*}{0.05} \\
\hline$>1000$ & $45(16 \%)$ & $243(84 \%)$ & \\
\hline \multicolumn{4}{|l|}{ Tumor budding } \\
\hline Positive & $28(26 \%)$ & $78(74 \%)$ & \multirow[t]{2}{*}{$<0.01$} \\
\hline Negative & $18(8 \%)$ & $198(92 \%)$ & \\
\hline \multicolumn{4}{|c|}{ Tumor differentiation } \\
\hline Well & $23(9 \%)$ & $225(91 \%)$ & \multirow[t]{2}{*}{$<0.01$} \\
\hline Moderate/poor & $23(31 \%)$ & $51(69 \%)$ & \\
\hline \multicolumn{4}{|c|}{ Lymphatic invasion } \\
\hline Positive & $25(33 \%)$ & $51(67 \%)$ & \multirow[t]{2}{*}{$<0.01$} \\
\hline Negative & $21(9 \%)$ & $225(91 \%)$ & \\
\hline \multicolumn{4}{|l|}{ Venous invasion } \\
\hline Positive & $13(29 \%)$ & $32(71 \%)$ & \multirow[t]{2}{*}{$<0.01$} \\
\hline Negative & $33(12 \%)$ & $244(88 \%)$ & \\
\hline
\end{tabular}

${ }^{\text {a }}$ Preserved or incompletely disrupted muscularis mucosa.

${ }^{\mathrm{b}}$ Completely disrupted muscularis mucosa.

Table 2 Multivariate analysis of risk factors for lymph node metastasis

\begin{tabular}{lccc}
\hline Factors & Odds ratio & $95 \%$ CI & P-value \\
\hline $\begin{array}{l}\text { Lymphatic invasion (+) vs } \\
\text { lymphatic invasion (-) }\end{array}$ & 3.19 & $0.22-0.94$ & $<0.01$ \\
$\begin{array}{l}\text { Well differentiation vs moderate/ } \\
\text { poor differentiation }\end{array}$ & 3.02 & $0.20-0.90$ & $<0.01$ \\
$\begin{array}{l}\text { Tumor budding (+) vs } \\
\text { tumor budding (-) }\end{array}$ & 2.59 & $0.12-0.83$ & $<0.01$ \\
\hline
\end{tabular}

patients who showed lymph node metastasis. Many investigators have reported that submucosal invasion depth is a risk factor for lymph node metastasis. ${ }^{1-7}$ However, problems exist with measurement of the depth of submucosal invasion when the muscularis mucosa is completely disrupted by tumor invasion. In such cases, measured submucosal invasion depths might be inconsistent. In this study, we were able to select patients with lymph node metastasis without measuring the submucosal invasion depth.

We propose that patients with submucosal invasive colorectal carcinoma should undergo additional treatment after endoscopic mucosal resection when the lesions show at least one of the following findings: moderate or poor differentiation, lymphatic invasion, tumor budding, or a type B status of the muscularis mucosa. If our current pathological 
criteria had been applied, 285 of the present 322 patients, including all 46 patients with lymph node metastasis, would have undergone additional colectomy after endoscopic mucosal resection. Thus, the sensitivity would be $100 \%$, whereas the specificity would be $13.4 \%$. The choice of threshold should depend primarily on the purpose of the overall clinical scheme; some investigators may require a higher sensitivity for clinical application, while sacrificing specificity, whereas others may choose the opposite. In this study, the sensitivity was $100 \%$, which can be regarded as sufficient for use as a screening test, at the expense of low specificity.

In 2005, we reported the results of uni- and multivariate analyses to identify histopathological factors associated with lymph node metastasis in 155 patients with submucosal invasive colorectal carcinoma who underwent colectomy with lymph node dissection. ${ }^{6}$ Multivariate analysis showed that moderate or poor tumor differentiation, lymphatic invasion, and high-grade focal dedifferentiation, namely tumor budding at the invasive front, were independently associated with lymph node metastasis. In this study, moderate or poor tumor differentiation, lymphatic invasion, and tumor budding at the submucosal invasive front were confirmed to be independently correlated with lymph node metastasis by multivariate analysis using a larger population of patients with submucosal invasive colorectal carcinoma.

In this analysis, all the patients underwent colectomy with lymph node dissection, resulting in the sampling of a large number of lymph nodes: 1-63 (median 14). Most previous reports have analyzed only small numbers of dissected lymph nodes: $7.3 \pm 4.9$ lymph nodes in the study by Nascimbeni et $a l,{ }^{3}$ and a median number of 10 in the study by Okabe et $a l,{ }^{5}$ whereas the number of removed lymph nodes was not described in several other reports. ${ }^{4,6,7}$ Wang et $a l^{12}$ showed that the total number of sampled lymph nodes was associated with the prognosis of submucosal invasive colorectal carcinoma. Therefore, accurate diagnosis of lymph node status by retrieving a sufficient number of nodes is essential for examining the relationship between clinicopathological factors and lymph node metastasis. A median of 14 lymph nodes were evaluated in our present study, which was the largest number examined in any other reports published so far. In addition, in our study, the patients had been treated using the same therapeutic procedure, ie, colectomy with lymph node dissection, and the resected specimens were examined in the same manner at a single institution. Previous studies of the association between histopathological factors and lymph node metastasis have involved series of 129-353 cases, ${ }^{1-3,5-7}$ except for one in which 865 cases from six institutions were examined. ${ }^{4}$ Thus, our study used one of the largest reported series of lymph node metastasis cases in patients with submucosal invasive colorectal carcinoma examined at a single institution.

Surgically resected specimens are usually cut into 5-mm-thick sections, whereas endoscopic mucosal resection specimens are cut into 2-mm-thick serial sections. Therefore, endoscopic mucosal resection specimens are evaluated more meticulously than surgically resected specimens, allowing predictive factors for lymph node metastasis to be detected more frequently. From this viewpoint, our current data obtained from surgically resected specimens should be safely applicable to endoscopic mucosal resection specimens.

\section{Disclosure/conflict of interest}

The authors declare no conflict of interest.

\section{References}

1 Haggitt RC, Glotzbach RE, Soffer EE, et al. Prognostic factors in colorectal carcinomas arising in adenomas: implications for lesions removed by endoscopic polypectomy. Gastroenterology 1985;89:328-336.

2 Coverlizza S, Risio M, Ferrari A, et al. Colorectal adenomas containing invasive carcinoma. Pathologic assessment of lymph node metastatic potential. Cancer 1989;64:1937-1947.

3 Nascimbeni R, Burgart LJ, Nivatvongs S, et al. Risk of lymph node metastasis in $\mathrm{T} 1$ carcinoma of the colon and rectum. Dis Colon Rectum 2002;45: 200-206.

4 Kitajima K, Fujimori T, Fujii S, et al. Correlations between lymph node metastasis and depth of submucosal invasion in submucosal invasive colorectal carcinoma: a Japanese collaborative study. J Gastroenterol 2004;39:534-543.

5 Okabe S, Shia J, Nash G, et al. Lymph node metastasis in T1 adenocarcinoma of the colon and rectum. J Gastrointest Surg 2004;8:1032-1039; discussion 1039-1040.

6 Tominaga K, Nakanishi Y, Nimura S, et al. Predictive histopathologic factors for lymph node metastasis in patients with nonpedunculated submucosal invasive colorectal carcinoma. Dis Colon Rectum 2005;48:92-100.

7 Egashira Y, Yoshida T, Hirata I, et al. Analysis of pathological risk factors for lymph node metastasis of submucosal invasive colon cancer. Mod Pathol 2004;17:503-511.

8 Kudo S. Endoscopic mucosal resection of flat and depressed types of early colorectal cancer. Endoscopy 1993;25:455-461.

9 Stanley RH, Lauri AA. World Health Organization Classification of Tumors. IARC Press: Lyon, France, 2000.

10 Ueno H, Mochizuki H, Hashiguchi Y, et al. Risk factors for an adverse outcome in early invasive colorectal carcinoma. Gastroenterology 2004;127:385-394.

11 Ueno H, Murphy J, Jass JR, et al. Tumor 'budding' as an index to estimate the potential of aggressiveness in rectal cancer. Histopathology 2002;40:127-132.

12 Wang HS, Liang WY, Lin TC, et al. Curative resection of T1 colorectal carcinoma: risk of lymph node metastasis and long-term prognosis. Dis Colon Rectum 2005;48:1182-1192. 